\title{
Injury of the basilar artery associated with closed head trauma ${ }^{1}$
}

\author{
CHENG-MEI SHAW AND ELLSWORTH C. ALVORD, JR. \\ From the Laboratory of Neuropathology, Department of Pathology, \\ University of Washington Medical School, Seattle, Washington 98105, U.S.A.
}

SUMMARY The neuropathological findings at necropsy are described in three cases of basilar artery injuries and their consequences after head trauma and the mechanism of injuries is discussed. The first case was that of massive pontine infarct due to an occlusion of the basilar artery trapped in the fracture of the clivus, 13 days before death. The second case, also with a pontine infarct, survived for two and two-thirds months; the trauma was probably similar, but the presence of the basal skull fracture and the occlusion of the basilar artery can be surmised only in retrospect. The third case was one of delayed rupture of the basilar artery occurring three to four weeks after the injury, which caused aneurysmal formation in the left posterior cerebral artery.

Since blood vessels are components of most organs and tissues, vascular damage of various types is almost inevitable with trauma of any tissues. Epidural, subdural, and intracerebral haemorrhages are well-established clinical and pathological entities related to the laceration of the meningeal artery, superficial cortical veins, and smaller intracerebral vessels, respectively.

Traumatic internal carotid artery thrombosis is another entity more recently recognized. Other disorders include dissecting aneurysm, delayed rupture, false and true aneurysmal formation with or without rupture, and embolization; these occur infrequently and the recognition of these conditions is extremely difficult without careful necropsy studies. These vascular complications appear to be particularly rare in the posterior circulation of the brain involving the vertebral and basilar artery system.

In the following report, three cases that came to necropsy of basilar artery injuries associated with head trauma are presented. The mechanism of the injury in these cases was quite unusual. Two cases showed massive pontine infarct due to an occlusion of the basilar artery, in one case shown to be trapped in the fracture of the clivus. The radiographic and EEG findings in this case have been previously reported (Loop, White, and Shaw, 1964; Chatrian, White, and Shaw, 1964). A similar finding was not demonstrated in the

\footnotetext{
1 Supported in part by Neuropathology Training Grant 5 TO1 NSO 5231-13 from NINDS.
}

basilar artery in the second case but circumstantial evidence strongly suggests a similar type of injury. The third case represents an example of delayed rupture of the basilar artery and aneurysmal formation of left posterior cerebral artery probably due to overstretching of the blood vessels causing an intimal tear.

\section{CASE 1}

(Np 483). This 59 year old construction worker was admitted to the Emergency Room on 22 November 1961 after being struck on the forehead by a large falling timber 30 minutes previously. $\mathrm{He}$ fell to the ground, after the blow, unconscious with bleeding face.

On admission the patient was unresponsive except for decerebration of the arms on inspiration and when vomiting. There was a large ecchymosis and swelling on the forehead above the left eye, the nose was bleeding profusely and the pharynx was filled with blood clot. Both ears were also blood-filled. Neurologically, the patient had a trace of a doll's eye reflex (oculocephalic reflex), the right was slightly larger than the left, both reacted slightly to light, deep tendon reflexes were symmetrical, and Babinski's sign was present bilaterally. The arms were hypertonic, held in a decerebrate position and moved with painful stimulation. The legs showed much less tone and less evidence of decerebrate movement.

An endotracheal tube was inserted after admission. The blood pressure fluctuated between 140 and 110 $\mathrm{mm} \mathrm{Hg}$ systolic and between 80 and $70 \mathrm{~mm} \mathrm{Hg}$ diastolic. A lumbar puncture revealed an opening pressure of $130 \mathrm{~mm} \mathrm{CSF}$, with bloody fluid and a 
haematocrit of $2 \%$, glucose $96 \mathrm{mg} / 100 \mathrm{ml}$. and protein $52 \mathrm{mg} / 100 \mathrm{ml}$.

The patient's condition remained unchanged during the next few hours except for the development of random movements of the right eye with the left eye fixed in a neutral position and then development of random movements of both eyes in a disconjugate fashion. A tracheostomy was performed the next day.

The patient was treated with atropine, antibiotics, and other supportive measures. He continued to show decerebrate movements of arms and legs after painful stimulation. A spinal puncture repeated on day 5 revealed an opening pressure of $250 \mathrm{~mm} \mathrm{CSF}$ with cloudy xanthochromic fluid. EEG on the same day revealed a rhythm of 8 to $10 \mathrm{~Hz}$ and of 10 to $35 \mu \mathrm{V}$ which closely resembled the alpha rhythm of a waking subject (Chatrian et al. 1964). A right carotid arteriogram was performed on day 12 and showed partial filling of the proximal basilar artery and both posterior cerebral arteries (Loop et al. 1964).

The patient developed respiratory difficulty and died the next day, 13 days after the injury. Necropsy was performed by Dr. W. L. Taylor, who noted bilateral bronchopneumonia and purulent bronchitis.

NEUROPATHOLOGICAL FINDINGS. The temporal muscles appeared to be markedly haemorrhagic but not swollen. There was a linear fracture of the right temporal bone extending into the parietal bone. A thin blood clot was found on the surface of the dura over the right frontotemporal region immediately underneath this fracture. Difficulties were encountered during removal of the brain because of the attachment of the basilar artery to the base of the skull. A segment of basilar artery, therefore, was severed and was left in the base of the skull. Inspection of the base of the skull after removal of the brain revealed a linear but zigzag fracture of the clivus extending into the dorsum sellae. The proximal two-thirds of the basilar artery was caught in and pinched by this fracture (Fig. 1). The dorsum sellae was mobile and removed with ease. Dark semiliquid blood clot filled the sphenoid sinus.

The brain weighed $1,400 \mathrm{~g}$ in the fresh state. Mild subarachnoid haemorrhages were seen along a few sulci in the right parietal lobe and in both occipital lobes. There was no evidence of cerebral swelling. The subarachnoid spaces were dilated and contained large amounts of cerebrospinal fluid. There was no transtentorial or cerebellar herniation. There were practically no atherosclerotic changes of the major blood vessels. No anomaly was found in the circle of Willis. The distal $1.5 \mathrm{~cm}$ of the basilar artery was attached to the brain and the remainder of the artery was left in the skull as described. The base of the pons immediately beneath the missing basilar artery

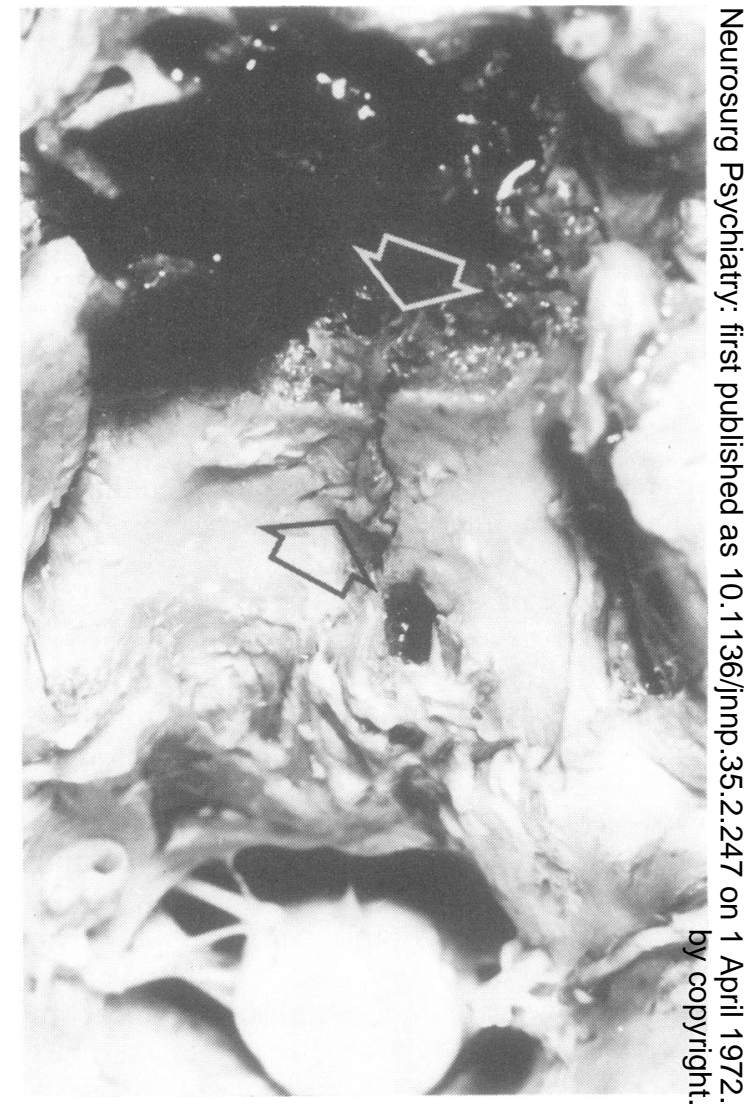

FIG. 1. The base of the skull in case 1. The dorsum sellae has been removed and the sphenoid sinus is filled with blood (top). Two arrows point to both ends of the trapped basilar artery in the fracture of the clivus.

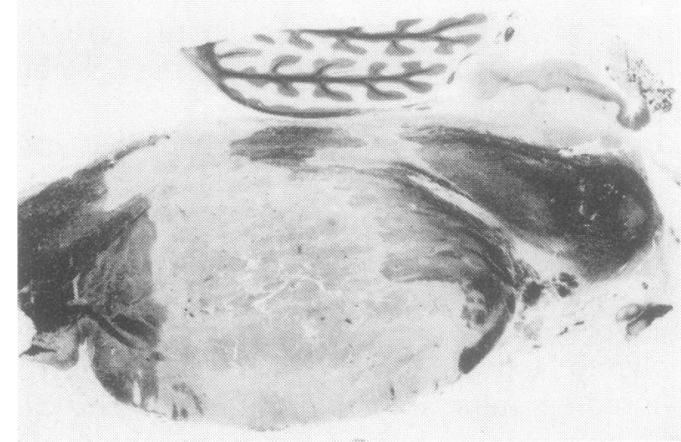

FIG. 2. A midsagittal section of the brain-stem in case 1 (LFB-PAS-H stain). Ischaemic infarct of the central pons, sparing both rostral and caudal ends, is shown by swelling and pallor. 
was slightly haemorrhagic and markedly swollen with softening which extended slightly more to the left side.

A midsagittal section of the brain revealed the entire cut section of the pons to be soft and slightly discoloured. The floor of the 4th ventricle was elevated by the swollen tegmentum. There was a small subarachnoid haemorrhage in the interpeduncular fossa extending into the parenchyma at the midbrain-pontine junction. Both midbrain and medulla were normal (Fig. 2).

The left half of the brain-stem was sectioned horizontally and the right half parasagittally, and the extent of the lesion was studied microscopically. The lesion was an ischaemic infarction, compatible with the clinical history of two weeks' duration, with abundant gitter cells and capillary proliferation at the rim of the lesion. The centre of the lesion was pale, without an increase of cells.

Horizontal sections of the left side of the brainstem revealed complete transection of the pons just above the level of the trigeminal nerve. In the rostral pons all of the tegmentum was destroyed, the lesion including the medial longitudinal fasciculus, locus caeruleus, lateral spinothalamic tract, and lateral lemniscus; the superior cerebellar peduncle was partially preserved but pale and swollen. The basis pontis was almost completely destroyed except for a very small peripheral island laterally. At the level of the trigeminal root, the lateral tegmentum was partially preserved, including a few of the most caudal cells of the locus caeruleus, but the mesencephalic trigeminal nucleus was destroyed by a still more lateral lesion involving the brachium pontis.

At the level of the abducens nucleus, the lateral tegmentum was spared completely, including the vestibular nuclei, solitary tract, nucleus parvocellularis, and the lateral part of the nucleus pontis centralis caudalis. The medial tegmentum was destroyed, including most of the nucleus gigantocellularis, the medial part of the nucleus pontis centralis caudalis, and the fibres of the abducens nerve in the ventral tegmentum. Dorsally, however, the abducens nucleus itself escaped, although the lesion coursed medially to the subependymal glia and destroyed the medial longitudinal fasciculus. The lesion expanded ventrally from this mediodorsal point through the medial tegmentum and into the basis pontis just lateral to the abducens fibres, and destroyed all but a small amount of the dorsolateral nuclei pontis immediately ventral to the spinal trigeminal tract.

More caudally, at the level of the facial and ventral cochlear nuclei, the tegmentum was normal, and there was only a small lesion in the pyramid. Still more caudally, in the medulla, no lesion was seen.

In the midsagittal and parasagittal sections of the right side of the brain-stem practically the entire basis pontis was destroyed except for a few milli- metres at each end. The tegmentum was, however, only partially destroyed medially by two tongue-like dorsal extensions of the massive basal lesion. Each of these tongues extended through the medial longitudinal fasciculus to the subependymal glial zone, but between these tongues was an island of dorsomedial tegmentum and central tegmental tract in the central pons. The rostral lesion included the caudal medial fibres of the oculomotor nerve at their point of exit and curved dorsally and caudally to destroy the caudal tip of the locus caeruleus. Laterally, however, this rostral lesion rapidly disappeared so that the mesencephalic and rostral pontine tegmentum remained intact. The caudal lesion was just at the level of the abducens nucleus and its nerve, and appeared to split these structures, destroying the rostral half and sparing the caudal half. The caudal edge of this lesion was relatively flat, but the rostral edge tapered laterally, resembling the rostral tongue-like lesion.

INTERPRETATION AND SUMMARY. The patient was probably looking up when the timber fell on his forehead and face. The whole head was compressed against the spinal column at the base, specifically at the clivus, which split with its overlying dura mater exactly beneath the basilar artery. The basilar artery fell into the fracture line and was trapped when the deformed skull sprang back to its normal shape and closed the fracture line. The basilar artery was instantly occluded, resulting in a massive pontine infarct which extended from the caudal end of the central tegmentum of the midbrain to the pontomedullary junction. The lateral and dorsal tegmentum of the midbrain and the medulla, except for a narrow extension, was spared. The pons was completely functionally transected on the left side at, or just above, the level of the trigeminal nerve; however, the tegmental lesion was incomplete on the right side.

\section{CASE 2}

(Np 2337). This was a 47 year old man who was involved in a head-on-collision automobile accident on 27 October 1967. The patient was the driver and became unconscious from the onset of the accident. He was taken to a hospital where he had respiratory and cardiac arrest and was resuscitated. He was in coma with decerebrate rigidity, fracture of the left frontal bone, and comminuted fracture of the right femur. Tracheostomy and gastrostomy were performed, and he gradually improved. He appeared to be somewhat aware of his surroundings three weeks later, but was unable to swallow and unable to move his extremities except his left upper limb to painful stimuli. The right femoral fracture was internally reduced on the sixth day and he was transferred to the Seattle Veterans Administration Hospital one month after the accident.

On admission he was able to follow command 
with his eyes and blinked his eyes on command. There was no reaction to threat to the eyes. Ocular movement was better to the right side. The left pupil was $2 \mathrm{~mm}$ larger than the right and both reacted sluggishly to light. The left corneal reflex was depressed. Left facial palsy was present. There was no cough or gag reflex. No movement of the extremities was noted. Deep tendon reflexes were hyperactive bilaterally except for the right knee jerk, which was absent. Babinski's sign was present bilaterally.

After his admission he developed high fever and jaundice. The diagnosis of hepatitis was made. He also developed massive upper gastrointestinal haematemesis two months after injury and was treated with blood transfusions. Intermittent bleeding continued and he finally died 11 weeks after the injury. Necropsy was performed by Dr. R. Tank, who reported acute coronary occlusion with myocardial infarct, haemorrhagic glomerulonephritis and probably serohepatitis.

NEUROPATHOLOGICAL FINDINGS The brain showed old irregular cortical contusions over the medial orbital frontal gyri bilaterally with orange discolouration. The lesions were predominantly on the right side, involving the gyrus rectus and extending from the frontal pole to the olfactory trigone. The olfactory bulbs and tracts could not be identified. The rest of the leptomeninges were unremarkable except for a small area over the inferior surface of the right cerebellar hemisphere, where a yellow tinge was present. The blood vessels showed no atherosclerotic changes, but the basilar and vertebral arteries were not present.

Coronal sections of the cerebrum revealed no lesion except for necrosis and atrophy of the right hippocampus and cortical defects of the orbital frontal gyri corresponding to the contusions noted externally. The ventricles were of normal size.

Horizontal sections of the brain-stem and cerebellum revealed a large irregular cystic necrosis in the basis of the caudal pons. The cyst of the basis was slightly larger on the left. The lesion began at the level of the trigeminal roots cranially and ended at the rostral medulla. At the level of the trigeminal roots there were bilaterally symmetrical, pinhead size, cystic areas of necrosis in the region of the facial nuclei. At the level of the abducens nuclei the necrosis extended up dorsally to involve both abducens and facial nuclei on the left side. A small patchy necrosis in the right medial tegmentum transected the abducens nerve.

At the level of the brachium pontis, the basis pontis was completely occupied by a cyst except for a ventral peripheral rim of $4 \mathrm{~mm}$ thickness (Fig. 3). A layer of loose necrotic tissue consisting of gitter cells, capillaries, and glial trabecula surrounded the empty cyst. Hypertrophic astrocytes were present in the outer rim of this necrotic layer and extended into

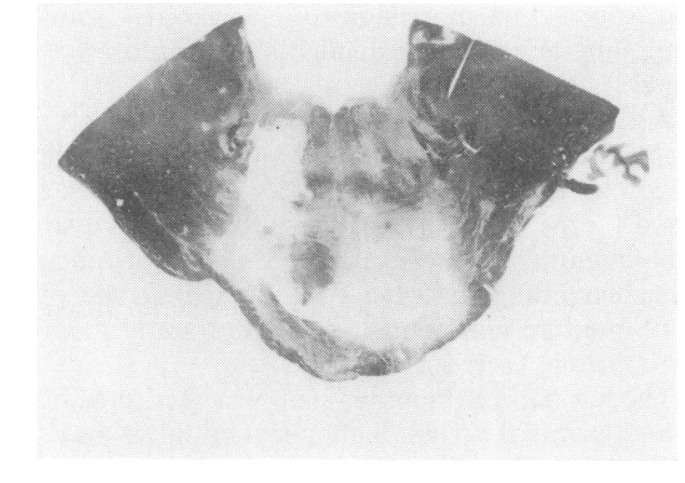

FIG. 3. A horizontal section of the midpons in case $2 \stackrel{2}{0}$ (LFB-PAS-H stain). Cystic necrosis involves the basis ${ }^{\circ}$ pontis and extends into the tegmentum on the left side. $\vec{O}$

the adjacent tissue, which appeared spongy in some places. Peripheral extensions of the necrosis in the ${ }_{\omega}$ tegmentum were frequently not cystic but filled with gitter cells, capillaries, and loose glial trabecula. The

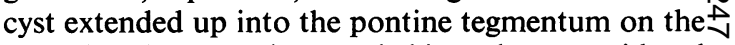
left side. The necrosis extended into the pyramid ando supraolivary region of the rostral medulla on tberight side.

INTERPRETATION AND SUMMARY In this case, fracture in the clivus with trapping of the basia artery was not anatomically demonstrated. The? prosector recalled that the brain was stuck at the base of the skull at the time of the removal ands accounted for the absence of the basilar and verte-O bral arteries on that basis. Unfortunately, however, careful observations of the base of the skull were noto made and the missing arteries were not searched for $\overrightarrow{\vec{O}}$ at the time of necropsy.

The principal lesion was in the caudal half of the pons and mainly involved the basis pontis with? patchy and finger-like extensions into the medioventral tegmentum, more so on the left side. The lesion was undoubtedly the result of ischaemiconecrosis, specifically due to an occlusion of the caudal half of the basilar artery. The lateral teg-ô mentum of the corresponding level of the pons was spared because its arterial supply came from theo large circumferential arteries, the superior and inferior cerebellar arteries, supplied rostrally ando caudally to the occluded middle part of the basilar artery. The patient's quadriplegia and pseudobulbaros palsy were due to almost total destruction of cortico- $N$ spinal and corticobulbar tracts. His wakefulness probably related to the preservation of the trigeminal ${ }_{\sigma}$ input and its connections with the upper reticular formation.

Haemorrhagic lesions of the tegmentum of the upper brain-stem due to axial displacement fre $\rightarrow$ 
quently following transtentorial herniation of the medial temporal lobes played no role in this case.

The necrosis and atrophy of the right hippocampus was probably due to insufficiency of the right posterior cerebral artery and its long branches of Uchimura (Lindenberg, 1955).

\section{CASE 3}

(Np 2687). This 30 year old Indian male was in a motorcycle accident in Alaska on 31 July 1968, but no details of the accident were known. The patient was found semiconscious with the odour of alcohol on his breath. He was bleeding from the nose and mouth and also vomited blood. He responded to painful stimuli, talked incoherently, and was able to move all extremities. The patient was transferred to the U.S. Public Health Service Hospital the next morning.

At the time of physical examination he was obtunded and disoriented; he responded to tactile and verbal stimuli but sometimes inappropriately. The blood pressure was $130 / 70 \mathrm{~mm} \mathrm{Hg}$ and the pulse 76 per min and regular. There were bilateral periorbital ecchymoses, left more severe than the right, dried blood in the external nares, left conjunctival haemorrhage but no other evidence of external trauma to the head. The pupil was slightly larger on the right but both reacted to light. He moved all extremities. Deep tendon reflexes were slightly hyperactive with bilateral ankle clonus but no pathological reflex was present. The radiograph of the skull showed a frontal fracture with possible orbital extension on the right side.

The patient remained stable, easily arousable but drowsy. On the second day there was left facial palsy and slurred speech. He was transferred to Seattle on the third day because his sensorium failed to clear. The physical examination at this time showed the patient to be lethargic, responding to verbal stimuli, but confused. The pupils were equal and reacted to light. There was an incomplete left facial palsy, left conjunctival haemorrhage, absent corneal reflexes bilaterally, neck rigidity, unsustained ankle clonus, positive Gonda reflex on the left, and slightly hyperactive deep tendon reflexes.

Antibiotics were started because the patient had a mild fever. A right carotid arteriogram was performed on the fourth day and showed no shift or other lesion. A lumbar puncture showed initial pressure of $180 \mathrm{~mm}$ of CSF, bloody CSF with a haematocrit of $1 \%$ and xanthochromic supernatant fluid. On the fifth day Ritalin was given but was discontinued because the patient became markedly hyperactive. The patient remained febrile for the next four days. On the ninth day his left facial palsy became complete, and the left facial nerve was decompressed. At this exploration the fracture was noted to extend through the distal edge of the petrous bone and to run across the geniculate ganglion. On the tenth day the temperature was $40.5^{\circ} \mathrm{C}\left(105^{\circ} \mathrm{F}\right)$ but cultures from blood, CSF, sputum, and urine were all negative. A repeat lumbar puncture next day showed initial pressure of $160 \mathrm{~mm}$ CSF and slightly xanthochromic fluid, and the day after that antibiotics were discontinued. The serum sodium was $125 \mathrm{~m}$-equiv/l. and the urinary volume was 91 . Two days later left hemiparesis was noted but another lumbar puncture showed initial pressure of $160 \mathrm{~mm}$ CSF.

On the 17th day the patient suddenly became more alert and oriented, sat up and began taking food by mouth. This condition lasted for about two days and he again began to deteriorate with fever persisting. The patient became progressively worse and comatose. On the 28th day lumbar puncture showed an initial pressure of $230 \mathrm{~mm}$ of CSF, which was xanthochromic; $20 \mathrm{ml}$. CSF was removed to reduce the pressure to $120 \mathrm{~mm}$ water. A twist drill ventricular tap was performed showing a pressure 0 $360 \mathrm{~mm}$ CSF; $50 \mathrm{ml}$. CSF was removed and $20 \mathrm{ml}$. air was injected. The ventriculogram showed a diffusely enlarged ventricular system. The patient deteriorated through the night and on the following morning he was deeply comatose with fixed dilated pupils. Repeated ventricular tap showed a pressure of $600 \mathrm{~mm}$ CSF. A ventriculostomy was inserted and tracheostomy was also performed. The ventricular drainage was found to be bloody with some clots. The patient became progressively worse and died on the afternoon of the same day, 29 days after the accident.

Necropsy was performed by Dr. R. Beagle, who noted a complicated stellate fracture of the left frontal bone, extending into the left anterior fossa, where the fracture line was about 1 in. to the left of the crista galli and more or less parallel to it. The fracture ran across the sphenoid bone, slightly to the left of the left anterior clinoid process, and then extended into the medial middle fossa, cutting the tip of the left petrous bone. It extended further into the posterior cranial fossa and ended near the foramen magnum.

NEUROPATHOLOGical Findings There was diffuse, recent and old, subarachnoid haemorrhage, particularly about the base of the brain, centred about the basilar artery and the circle of Willis, but also extending over both cerebral hemispheres. The brain was markedly and diffusely swollen. Its gyri were broad and its sulci were obliterated. There was marked tonsillar herniation. These was no atherosclerosis. Just proximal to the bifurcation of the basilar artery there was a round aneurysm, $1 \mathrm{~cm}$ in diameter, lying between the left posterior cerebral artery and the left superior cerebellar artery (Fig. 4). The aneurysm and the surrounding blood clot filled the interpeduncular fossa. The left third nerve 


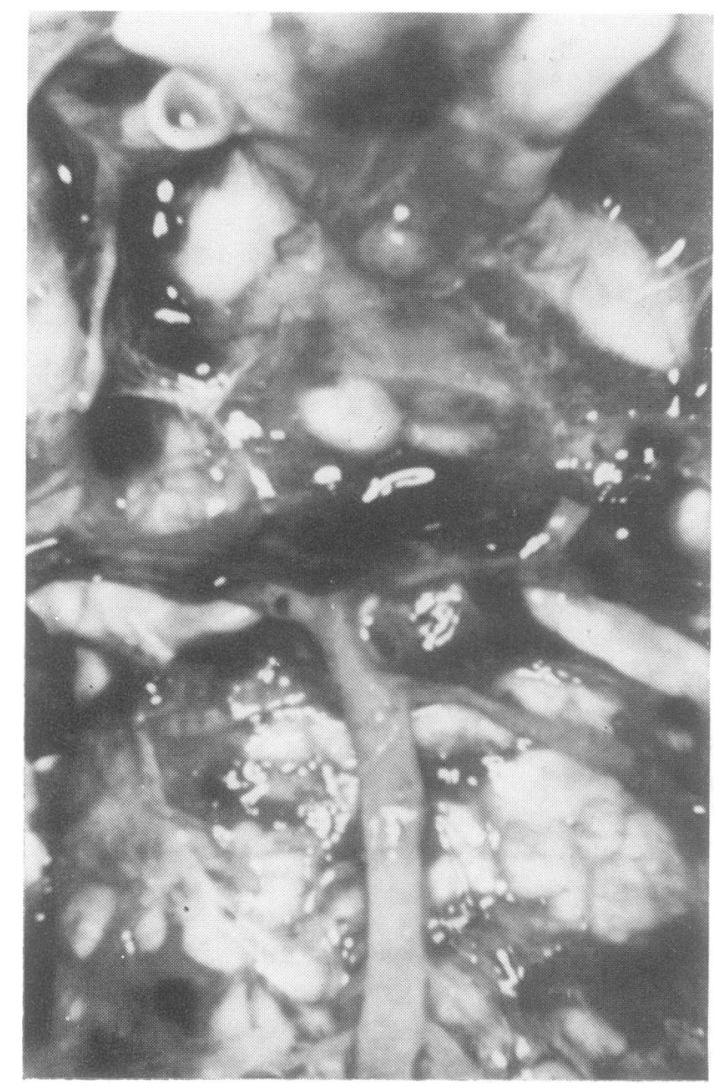

FIG. 4. The base of the brain in case 3. Most of the fresh subarachnoid blood has been removed. An aneurysm is attached to the left side of the distal basilar artery.

emerged from the lateral border of the aneurysm and was haemorrhagic.

On coronal sections of the cerebral hemispheres there was a $1 \mathrm{~mm}$ punctate haemorrhage in the left parolfactory area just beneath the anterior commissure. Several needle tracts were present bilaterally in the superior frontal gyri. The cavum septi pellucidi was only $1 \mathrm{~mm}$ across. Several small clots of blood were present in the right lateral ventricle. There was a granular brown discolouration and softening of the anterior portion of the left lingual gyrus and of the entire left fusiform and hippocampal gyri extending to the tip of the left temporal horn.

Horizontal sections of the brain-stem showed numerous small necrotic lesions in the median raphe of the midbrain and rostral pons and haemorrhagic necrosis in the periaqueductal grey matter of the rostral pons, left brachium conjunctivum, and floor of the 4th ventricle, more on the left side. The 4th ventricle contained blood. The cisterna ambiens was also filled with blood. The cerebellum showed no other lesion.

Serial microscopic sections of the distal basilaw artery including a short segment of the posterio cerebral and superior cerebellar arteries revealed ano additional small saccular aneurysm arising from the left posterior cerebral artery. Both aneurysms werê quite unusual, unlike those of the usual saccula aneurysm in many aspects. Both aneurysms arose. not at a crotch of the arteries but from the side of the arterial wall. There was a sudden interruption of the internal elastic membrane, as seen in the usuas saccular aneurysm, but the media did not disappea abruptly; instead, it gradually blended into a nodula fibroblastic scar involving the whole aneurysmaP wall. The aneurysmal sac then was made up of as crescent-shaped, partially organized blood clot. $A_{\vec{b}}$ very thin, fibrous tissue membrane was continuousfrom the adventitia of the mother artery and wentw around the blood clot, covered it only partially ans. then disappeared (Fig. 5). In other words, this aneurysmal sac was formed mainly by a partially organized blood clot and partly by a nodular thicks granulation tissue, which seemed to be continuous from the media of the mother artery.

On the other hand, the basilar artery aneurysmo did not contain any granulation tissue in its wall; in fact, it possessed practically no fibrous wall at all $>$ The aneurysm resembled a large round blood coge with partial organization, attached to a narrow gap of the basilar artery wall. The adventitia of the basifar artery continued to cover the clot for a short distance but then became unidentifiable. Both internal elasticas and media were disrupted at the gap (Fig. 6). In addition to these findings, there were several shorto segments of internal elastic membrane with multio focal areas of intimal'fibrosis near the gap in the

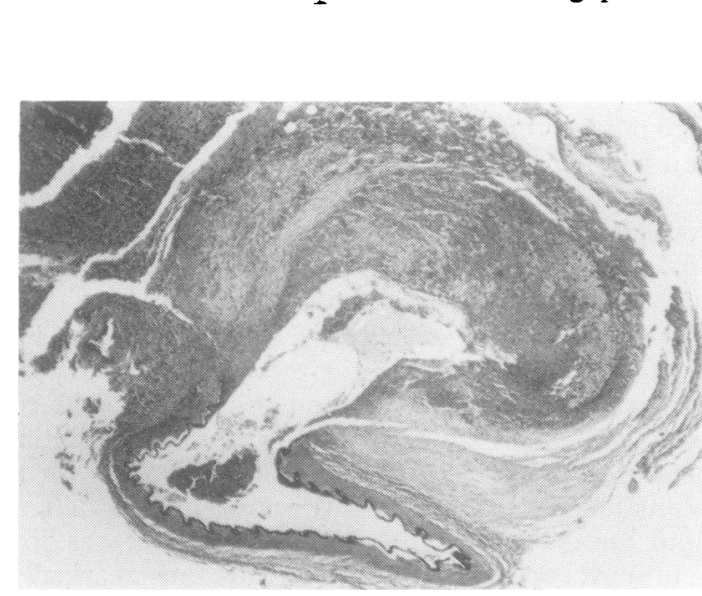

FIG. 5. Microscopic section of the aneurysm arising from the left posterior cerebral artery of case (Verhoff-Van Gieson stain). Granulation tissue and? organized blood clot form the sac of the aneurysm. 
arterial wall (Figs 7 and 8). Intimal fibrosis with occasional interruption of the internal elastica was not limited to the area near the gap of the artery but was found in many different segments of the basilar artery away from the aneurysm. The broken end of the internal elastica was at times pushed back and rolled into the lumen of the artery (Fig. 9).

INTERPRETATION AND SUMMARY At the moment of the fracture in the left side of the base of the skull, bony edges were displaced to pull or stretch the

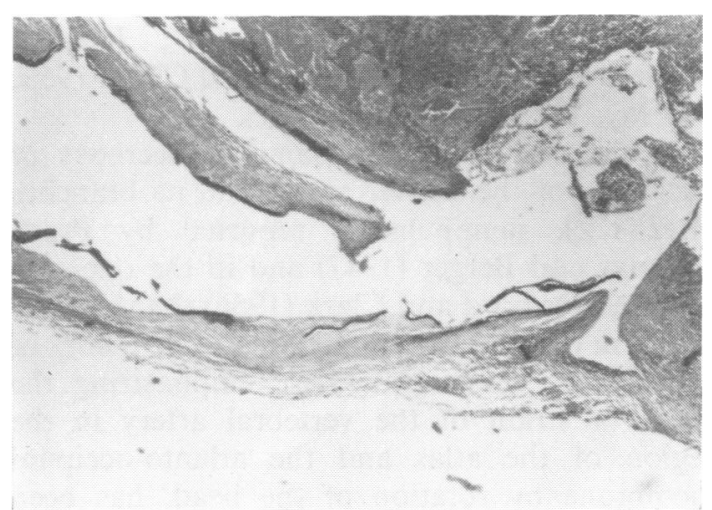

FIG. 6. Microscopic section of the aneurysm arising from the distal basilar artery of case 3 (Verhoff-Van Gieson stain). The aneurysm is really an organized blood clot (top) at the defect of the wall of the basilar artery. The clot is only partially covered by a thin connective tissue sac, which is the extension of the adventitia of the basilar artery (lower right). arteries at the base of the brain. As a result, the internal elastic membrane of the arteries was torn at many places. In these places we observed four different types of histological lesions: (1) aneurysmal formation in the left posterior cerebral artery (Fig. 5); (2) delayed rupture of the basilar artery (Fig. 6); (3) fibrotic scar formation; and (4) tear and occlusion of small branches of the basilar artery causing multiple small infarcts in the brain-stem.

The severity of the tear, the accompanying focal haemorrhages at the site of the tear, the focal necrosis, the extent of the inflammatory reaction, and the later focal haemorrhages in the developing granulation tissue are some of the factors which probably determine the type of histological reactions.

\section{DISCUSSION}

Occlusion of the internal carotid artery and its tributaries is not an uncommon complication of closed craniocervical injuries. The occlusion may occur in the cervical portion of the artery (Verneuil, 1872; Greco, 1935; Cairns, 1942; Northcroft and Morgan, 1945; Caldwell and Hadden, 1948; Schneider and Lemmen, 1952; Clarke, Dickson, and Smith, 1955; Sedzimir, 1955; Murray, 1957; Hockaday, 1959; Humphrey and Newton, 1960; Gurdjian, Hardy, Lindner, and Thomas, 1963; Therkelsen and Hornnes, 1963; Houck, Jackson, Odom, and Young, 1964; Toakley and McCaffrey, 1965; Pitner, 1966; Miller and Ayers, 1967; Hughes and Brownell, 1968; Verbiest and Calliauw, 1969); or at the base of the skull (Hockaday,
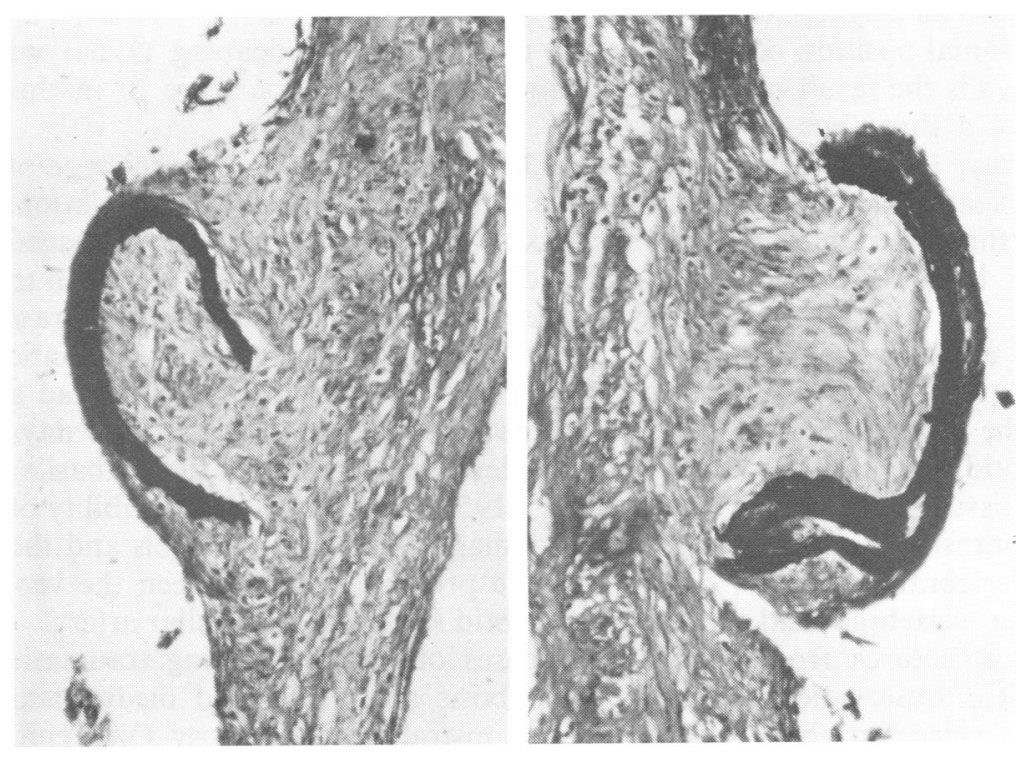

FIGS 7 and 8. Small segments of torn internal elastic lamina are present with focal scars in many areas of the wall of the basilar artery away from the aneurysm. VerhoffVan Gieson stain. 
1959, Thomson, 1963; Mastaglia, Savas, and Kakulas, 1969); or in the intracranial portion of the artery or its branches (de Veer and Browder, 1942; Dratz and Woodhall, 1947; Sedzimir, 1955; Shaw and Foltz, 1968). The occlusion can occur with or without a fracture of the base of the skull; but, when a basal skull fracture occurs, the site of the occlusion tends to be at the base of the skull or in the carotid canal (Sedzimir, 1955; Thomson, 1963; Houck et al. 1964).

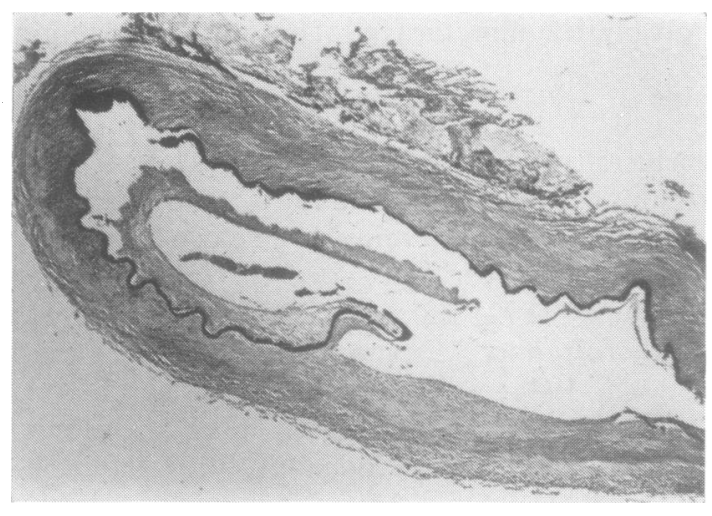

FIG. 9. A cross-section of the basilar artery proximal to the aneurysm shown in case 3 (Verhoff-Van Gieson stain). Focal defect of the internal elastica lamina, intimal and subintimal fibrosis and protrusion and retraction of internal elastic membrane are shown.

Some investigators have shown or suggested that the occlusion in the intracranial position of the carotid artery or its branches is the result of embolization or propagation of a thrombus in the neck (Sedzimir, 1955; Murray, 1957; Pitner, 1966; Miller and Ayers, 1967), and the possibility of propagation of the thrombus in the opposite direction has also been discussed (Sedzimir, 1955).

Similar sequences of events in the vertebrobasilar system are, however, quite infrequent. The rarity is partly due to the different anatomical relationships, the carotid artery in the neck being embedded in soft tissues and being more susceptible to a blunt contusion or overstretching, in contrast to the vertebral artery in the neck piercing through the vertebrae and being protected by the bony structures from contusion or stretching. Clinical observations are less frequently attempted, since vertebral arteriography is less commonly performed be- $\frac{0}{2}$ cause of technical difficulties and fewer indications than for carotid arteriography. Furthermore, postmortem removal of the cervical portion of the vertebral artery is also timeconsuming and not a routine procedure.

These are probably the major factors contribu- $=$ ting to the scarcity of reported cases of vertebral artery injury. Only a few cases of occlusion of the vertebral artery or its tributaries after离 trauma, cervical spine fracture, or chiropractico manipulation of the neck have been reported (Pratt-Thomas and Berger, 1947; Kunkle, $\frac{?}{\overrightarrow{3}}$ Muller, and Odom, 1952; Ford and Clark, 1956; $;$ Murray, 1957; Carpenter, 1961).

is

In the two cases that came to necropsy of $\vec{\circ}$ occlusion of the basilar artery and its branches after neck manipulation reported by Pratt- $\vec{\omega}$ Thomas and Berger (1947) and in the one case reported by Ford and Clark (1956) the vertebralo artery in the neck was not studied. The theory of Kleyn and Versteegh (1933), emphasizing theiv point of strain of the vertebral artery in the region of the atlas and the atlanto-occipital ${ }_{\odot}$ membrane by rotation of the head, has been $n_{-}^{\text {S }}$ discussed. Only in the case of Carpenter (1964) $\vec{D}$ was the vertebral artery in the neck found to geo necrotic with an intimal tear which produced $\overrightarrow{0}$ the propagating clot which extended into tân left posterior inferior cerebellar artery.

Only two cases have been reported of directo trapping of the basilar artery in the fracture of the clivus-our case 1 and the case of Sightso (1968). To the one case involving a similar directo trapping of the intracranial portion of the verte $-\overrightarrow{\vec{*}}$ bral artery reported by Lindenberg (1966) we now add another possible one (case 2) in this report.

Our case 3 illustrates a quite different type of injury to the arteries of the posterior circulation. The basal skull fracture ran near the damaged; arteries but did not appear to be close enough to ${ }^{3}$. cause a direct laceration of the artery. Brasso (1957) reported a case of indirect traumatic rupture of the basilar artery. His patient had a 9 fracture in the anterior fossa and died 16 days after the accident due to rupture of the basilar artery. Brass (1957) brought up the possibility of nutritional damage of the blood vessels and then

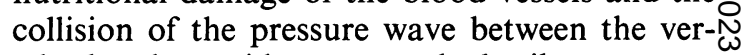
tebral and carotid system at the basilar artery. 응 In many cases of non-penetrating traumatico carotid thrombosis an intimal and medial tear has been demonstrated at necropsy (Verneuil, $\stackrel{?}{+}$ 
1872; Greco, 1935; Northcroft and Morgan, 1944; Caldwell and Hadden, 1948; Clarke et al. 1955; Murray, 1957). In those cases where direct contusion of the artery was not present, the mechanism of the intimal and medial injury has been attributed to overstretching or sudden torsion of the artery. An injury of similar mechanism undoubtedly occurs in the major intracranial arteries, but less frequently so, for the obvious reason that the mobility of the brain and blood vessels is much more limited within the cranial cavity. This limitation of the mobility may be violated by a transient displacement of the bony edges of a fracture. Therefore, the blood vessels may be overstretched, resulting in a selective laceration of the intima and media, which may also occur as the result of a direct contusion by displaced bony fragments or by the fibrous edges of the falx or tentorium.

The tear of the intima and media by these injuries could result in thrombus formation, dissecting aneurysm with occlusion of the arterial lumen, saccular aneurysmal formation, or immediate or delayed rupture of the artery. Most of the so-called 'post traumatic delayed apoplexy' cases are probably due to these lesions, most of which involve the carotid artery system, especially at its most proximal portion in the cranium, although the peripheral branches of the carotid artery system can be involved. Bots and Kramer (1964) described four cases of basilar artery thrombosis after severe acute craniocerebral injury dying five to nine days after the injury. They were able to demonstrate intimal damage in three and a microaneurysm in the basilar artery in one of these cases. In all of these cases, severe cerebral oedema and vascular stasis were present and it appears that these patients succumbed before 'delayed rupture' of the artery could take place.

As shown by experiments in dogs by Winternitz, Thomas, and LeCompte (1938) and in human necropsies by Glynn (1940), and summarized by Smith and Windsor (1961), the principal strength of the intracranial and cervical portions of the carotid artery is in different layers. In the intracranial artery the resistance of the arterial wall against the intraluminal pressure rests on the intimal internal elastic lamina, but the strength of the cervical portion of the carotid artery depends mainly on the adventitial elastic tissues. Thus, if a tear occurs in the intima, more precisely in the internal elastic layer, the consequences of the injury may be quite different depending on the site at which the artery is injured.

We agree with previous workers that laceration of the intima and media of the artery with preservation of the adventitia occurs by stretching and blunt contusion of the artery. We postulate that, when the intimal and medial tear occurs in the extracranial artery, in which the strength of the arterial wall is in the adventitial elastic tissue rather than in the internal elastica, occlusion of the artery after thrombus formation or dissecting aneurysm is the rule. Delayed rupture of the artery or formation of a saccular aneurysm in the neck after non-penetrating injury of the carotid artery practically never occurs. On the other hand, when the intimal and medial damage occurs in the intracranial arteries, where the principal strength of the arterial wall is in the internal elastica lamina, and where the adventitial elastic tissue is poorly developed, delayed rupture and saccular aneurysmal formation can also develop in addition to the occlusion of the lumen by dissecting aneurysm or thrombus formation.

Trauma is considered to be only a precipitating factor in the usual intracerebral aneurysm (Walton, 1956; Locksley, 1966), except in a few cases in which the direct relationship between the trauma and the aneurysmal rupture has been shown (Birley, 1928; Hirsch, David, and Sachs, 1962; Burton, Velasco, and Dorman, 1968; Smith and Bardenheier, 1968). The degeneration of the internal elastica in most cases is known to be caused by atherosclerosis (Forbus, 1930; Carmichael, 1950), but an intimal tear could occur after a relatively minor head injury and remain there silently for sometime until the person develops high blood pressure or until the damaged arterial wall serves as a locus minoris resistentiae for the development of a focal atheroma. In this remote way, trauma may contribute to the development of ordinary saccular aneurysms.

\section{REFERENCES}

Birley, J. L. (1928). Traumatic aneurysm of the intracranial portion of the internal carotid artery. With a note by $W$. Trotter. Brain, 51, 184-208.

Bots, G. Th. A. M., and Kramer, W. (1964). Traumatic thrombosis of intracranial arteries and extensive necrosis of the brain developed during reanimation. Acta Neuropathologica, 3, 416-427.

Brass, K. (1957). Uber indirekte traumatische Rupturen der Hirnbasisarterien. Frankfurter Zeitschrift für Pathologie, 68, 254-260. 
Burton, C., Velasco, F., and Dorman, J. (1968). Traumatic aneurysm of a peripheral cerebral artery. Review and case report. Journal of Neurosurgery, 28, 468-474.

Cairns, H. (1942). The vascular aspects of head injuries. Lisboa Médica, 19, 375-410.

Caldwell, H. W., and Hadden, F. C. (1948). Carotid artery thrombosis; report of eight cases due to trauma. Annals of Internal Medicine, 28, 1132-1142.

Carmichael, R. (1950). The pathogenesis of non-inflammatory cerebral aneurysms. Journal of Pathology and Bacteriology, 62, 1-19.

Carpenter, S. (1961). Injury of neck as cause of vertebral artery thrombosis. Journal of Neurosurgery, 18, 849-853.

Chatrian, G. E., White, L. E. jr., and Shaw, C.-M. (1964). EEG pattern resembling wakefulness in unresponsive decerebrate state following traumatic brain-stem infarct. Electroencephalography and Clinical Neurophysiology, 16 285-289.

Clarke, P. R. R., Dickson, J., and Smith, B. J. (1955). Traumatic thrombosis of the internal carotid artery following non-penetrating injury and leading to infarction of the brain. British Journal of Surgery, 43, 215-216.

de Veer, J. A., and Browder, J. (1942). Post-traumatic cerebral thrombosis and infarction. Report of a case and discussion of its bearing on the problem of immediate and delayed post-traumatic apoplexy. Journal of Neuropathology and Experimental Neurology, 1, 24-31.

Dratz, H. M., and Woodhall, B. (1947). Traumatic dissecting aneurysm of left internal carotid, anterior cerebral and middle cerebral arteries. Journal of Neuropathology and Experimental Neurology, 6, 286-291.

Forbus, W. D. (1930). On the origin of miliary aneurysms of the superficial cerebral arteries. Bulletin of the Johns Hopkins Hospital, 47, 239-284.

Ford, F. R., and Clark, D. (1956). Thrombosis of basilar artery with softenings in the cerebellum and brain-stem due to manipulation of the neck. Bulletin of the Johns Hopkins Hospital, 98, 37-42.

Glynn, L. E. (1940). Medial defects in the circle of Willis and their relation to aneurysm formation. Journal of Pathology and Bacteriology, 51, 213-222.

Greco, T. (1935): Le trombosi post-traumatiche della carotide. Archives Italiennes de Chirurgie, 39, 757-784.

Gurdjian, E. S., Hardy, W. G., Lindner, D. W., and Thomas, L. M. (1963). Closed cervical cranial trauma associated with involvement of carotid and vertebral arteries. Journal of Neurosurgery, 20, 418-427.

Humphrey, J. G., and Newton, T. H. (1960). Internal carotid artery occlusion in young adults. Brain, 83, 565-578.

Hirsch, J. F., David, M., and Sachs, M. (1962). Les anevrysmes artériels traumatiques intracraniens. Neurochirurgie, 8, 189-201.

Hockaday, T. D. R. (1959). Traumatic thrombosis of the internal carotid artery. Journal of Neurology, Neurosurgery, and Psychiatry, 22, 229-231.

Houck, W. S., Jackson, J. R. Odom, G. L., and Young, W. G. (1964). Occlusion of the internal carotid artery in the neck secondary to closed trauma to the head and neck. A report of two cases. Annals of Surgery, 159, 219-221.

Hughes, J. T., and Brownell, B. (1968). Traumatic thrombosis of the internal carotid artery in the neck. Journal of Neurology, Neurosurgery, and Psychiatry, 31, 307-314.

Kleyn, A. de, and Versteegh, C. (1933). Über verschiedene Formen von Ménières Syndrom. Deutsche Zeitschrift für Nervenheilkunde, 132, 157-189.

Kunkle, E. C., Muller, J. C., and Odom, G. L. (1952). Traumatic brain-stem thrombosis: report of a case and analysis of the mechanism of injury. Annals of Internal Medicine, 36, 1329-1335.

Lindenberg, R. (1955). Compression of brain arteries as pathogenetic factor for tissue necrosis and their areas of predilection. Journal of Neuropathology and Experimentat Neurology, 14, 223-243.

Lindenberg, R. (1966). Incarceration of a vertebral artery irf the cleft of a longitudinal fracture of the skull. Case reporto Journal of Neurosurgery, 24, 908-910.

Locksley, H. B. (1966). Report on the cooperative study of? intracranial aneurysms and subarachnoid hemorrhage Section V, Part I. Natural history of subarachnoid hemor< rhage, intracranial aneurysms and arteriovenous malforma $\frac{\rho}{?}$ tions, based on 6,368 cases in the cooperative study. Journa of Neurosurgery, 25, 219-239.

Loop, J. W., White, L. E. jr., and Shaw, C. M. (1964): Traumatic occlusion of the basilar artery within a clivus fracture. Radiology, 83, 36-40.

Mastaglia, F. L., Savas, S., and Kakulas, B. A. (1969). Intra음 cranial thrombosis of the internal carotid artery afteo closed head injury. Journal of Neurology, Neurosurgery, and $\overline{\widehat{D}}$ Psychiatry, 32, 383-388.

Miller, J. D. R., and Ayers, T. N. (1967). Post-traumatic changes in the internal carotid artery and its branches: an arteriographic study. Radiology, 89, 95-100.

Murray, D. S. (1957). Post-traumatic thrombosis of the internal carotid and vertebral arteries after non-penetratinginjuries of the neck. British Journal of Surgery, 44, 556-561.w

Northcroft, G. B., and Morgan, A. D. (1945). A fatal case of traumatic thrombosis of the internal carotid artery. British Journal of Surgery, 32, 105-107.

Pitner, S. E. (1966). Carotid thrombosis due to intraora trauma. An unusual complication of a common childhood accident. New England Journal of Medicine, 274, 764-767

Pratt-Thomas, H. R., and Berger, K. E. (1947). Cerebellarf and spinal injuries after chiropractic manipulation. Journal of the American Medical Association, 133, 600-603.

Raney, A. A. (1948). Cerebral embolism following mingrwounds of the carotid artery. Report of an autopsy. I Archives of Neurology and Psychiatry, 60, 425-439.

Schneider, R. C. and Lemmen, L. J. (1952). Traum internal carotid artery thrombosis secondary to nom- $\overrightarrow{0}$ penetrating injuries to the neck. A problem in the differe tial diagnosis of craniocerebral trauma. Journal of Neurosurgery, 9, 495-507.

Sedzimir, C. B. (1955). Head injury as a cause of internals carotid thrombosis. Journal of Neurology, Neurosurgery, and Psychiatry, 18, 293-296.

Shaw, C.-M., and Foltz, E. L. (1968). Traumatic dissectingڤ aneurysm of middle cerebral artery and carotid-cavernous fistula with massive intracerebral hemorrhage. Case report. Journal of Neurosurgery, 28, 475-479.

Sights, W. P., jr. (1968). Incarceration of the basilar artery in a fracture of the clivus. Case report. Journal of Neurosurgery, 28, 588-591.

Smith, D. E., and Windsor, R. B. (1961). Embryonic and pathogenic aspects of the development of cerebral saccular aneurysm. In Pathogenesis and Treatment of Cerebro-0 vascular Disease. Seventh Annual Scientific Meeting of the Houston Neurological Society, Texas Medical Center, Houston, Texas, pp. 367-397. Compiled and edited by" W. S. Fields. Thomas: Springfield, III.

Smith, K. R. jr., and Bardenheier, J. A. III (1968). Aneurysm of the pericallosal artery caused by closed cranial trauma.o Case report. Journal of Neurosurgery, 29, 551-554.

Therkelsen, J. and Hornnes, N. (1963). Traumatic occlusion of the internal carotid artery in a child. Restored circulation by means of thrombectomy. Circulation, 28, 101-104.

Thomson, J. L. G. (1963). Traumatic thrombosis of the internal carotid artery in the carotid canal. British Journaln of Radiology, 36, 840-842.

Toakley, G., and McCaffrey, J. (1965). Traumatic thrombosis of the internal carotid artery. Australian and New Zealand Journal of Surgery, 34, 261-264.

Verbiest, H., and Calliauw, L. (1959): Direct and indirect injuries of the cervical carotid arteries. A contribution to the differential diagnosis of the posttraumatic lucid interval. 
Folia Psychiatrica, Neurologica et Neurochirurgica, Neerlandica, 62, 371-382.

Verneuil (1872). Thrombose [de l'artère carotide.] Bulletin de l'Academie de Médicine de Paris, 2 me série, 1, 46-56, cited by Schneider and Lemmen and by Gurdjian et al.
Walton, J. N. (1956). Subarachnoid Haemorrhage. Livingstone : Edinburgh.

Winternitz, M. C., Thomas, R. M., and LeCompte, P. M. (1938). The Biology of Arteriosclerosis. Thomas: Springfield, Ill. 